\title{
Laboratory-Model Integrated-System FARAD Thruster
}

\author{
K.A. Polzin \\ NASA Marshall Space Flight Center \\ Huntsville, AL 35812 \\ S. Best \\ Space Research Institute, Auburn University \\ Auburn, AL 36849
}

\author{
M.F. Rose, R. Miller \\ Radiance Technologies \\ Huntsville, AL 35805
}

T. Owens

West Virginia High Technology Consortium

Foundation

Fairmont, WV 26554

\begin{abstract}
:
Pulsed inductive plasma accelerators are spacecraft propulsion devices in which energy is stored in a capacitor and then discharged through an inductive coil. The device is electrodeless, inducing a plasma current sheet in propellant located near the face of the coil. The propellant is accelerated and expelled at a high exhaust velocity (order of $10 \mathrm{~km} / \mathrm{s}$ ) through the interaction of the plasma current with an induced magnetic field.
\end{abstract}

The Faraday Accelerator with RF-Assisted Discharge (FARAD) thruster [1,2] is a type of pulsed inductive plasma accelerator in which the plasma is preionized by a mechanism separate from that used to form the current sheet and accelerate the gas. Employing a separate preionization mechanism in this manner allows for the formation of an inductive current sheet at much lower discharge energies and voltages than those found in previous pulsed inductive accelerators like the Pulsed Inductive Thruster (PIT).

In a previous paper [3], the authors presented a basic design for a $100 \mathrm{~J} /$ pulse FARAD laboratory-version thruster. The design was based upon guidelines and performance scaling parameters presented in Refs. [4,5]. In this paper, we expand upon the design presented in Ref. [3] by presenting a fully-assembled and operational FARAD laboratory-model thruster and addressing system and subsystem-integration issues (concerning mass injection, preionization, and acceleration) that arose during assembly. Experimental data quantifying the operation of this thruster, including detailed internal plasma measurements, are presented by the authors in a companion paper [6].

The thruster operates by first injecting neutral gas over the face of a flat, inductive acceleration coil and at some later time preionizing the gas. Once the gas is preionized current is passed through the acceleration coil, inducing a plasma current sheet in the propellant that is accelerated away from the coil through electromagnetic interaction with the time-varying magnetic field.

Neutral gas is injected over the face of the acceleration coil through a fast-acting valve that feeds a central distribution manifold. The thruster is designed to preionize the gas using an RFfrequency ringing signal produced by a discharging Vector Inversion Generator (VIG). The acceleration stage consists of a multiple-turn, multiple-strand spiral induction coil (see Fig. 1, left panel) and is designed for operation at discharge energies on the order of $100 \mathrm{~J} / \mathrm{pulse}$. Several different pulsed power train modules can be used to drive current through the acceleration coil. One such power train is based upon the Bernardes and Merryman circuit topology, which 
restricts voltage reversal on the capacitor banks and can be clamped to eliminate current reversal in the coil. A second option is a pulse-compression ring power train (see Fig. 1, right panel), which takes a temporally broad, low current pulse and transforms it into a short, high current pulse.

\section{REFERENCES}

[1] K.A. Polzin, Faraday Accelerator with Radio-frequency Assisted Discharge (FARAD), Ph.D. Dissertation, Princeton University, Princeton, NJ, 2006.

[2] E.Y. Choueiri and K.A. Polzin, "Faraday Acceleration with Radio-Frequency Assisted Discharge," Journal of Propulsion and Power, 22(3):611, 2006.

[3] K.A. Polzin, et al., "Design of a Low-Energy FARAD Thruster," in 43rd AIAA/ASME/ ASEE/SAE Joint Propulsion Conf., Cincinnati, OH, 2007. AIAA Paper 2007-5257.

[4] K.A. Polzin and E.Y. Choueiri, "Design rules for high-performance FARAD thrusters," in 29th International Electric Propulsion Conf., Princeton, NJ, 2005. IEPC Paper 2005-207.

[5] K.A. Polzin and E.Y. Choueiri, "Performance optimization criteria for pulsed inductive plasma acceleration," IEEE Transactions on Plasma Science, 34(3):945, 2006.

[6] K.A. Polzin, et al., "Operational characteristics and plasma measurements in a low-energy FARAD thruster," in 44th AIAA/ASME/SAE/ASEE Joint Propulsion Conf., Hartford, CO, 2008.
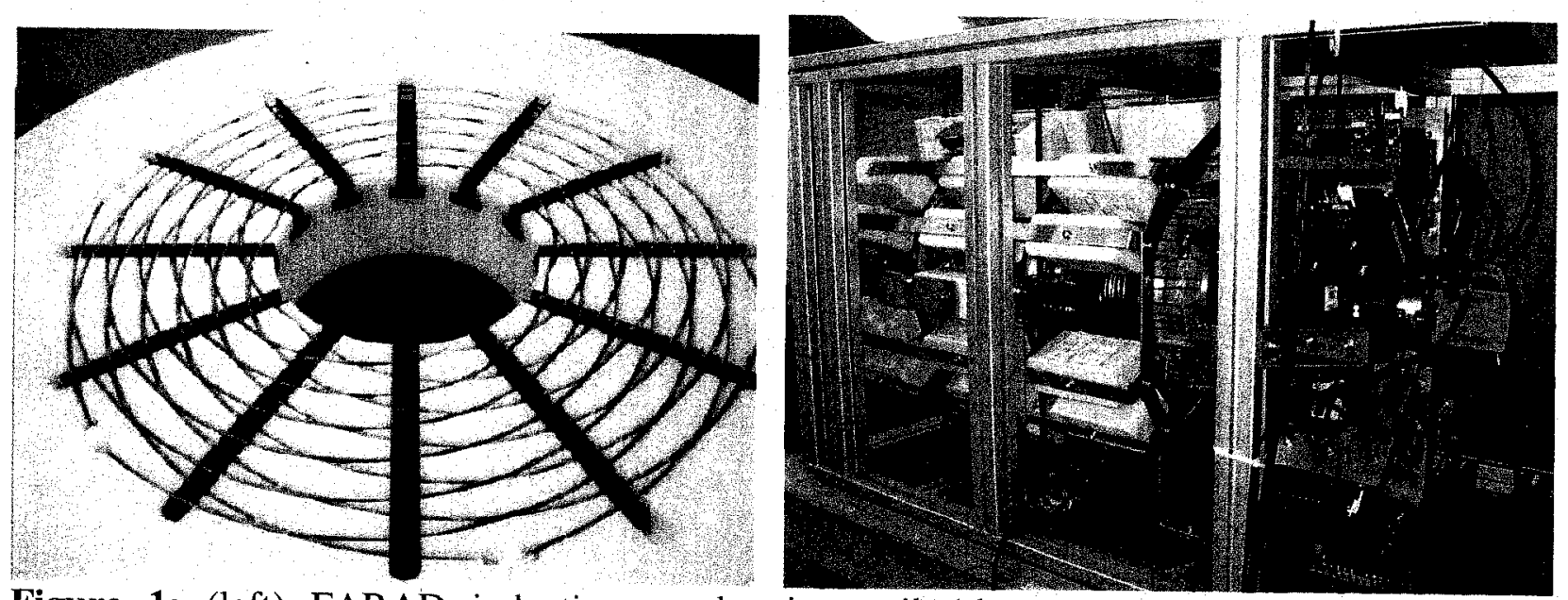

Figure 1: (left) FARAD inductive acceleration coil (shown unpotted) and (right) pulse compression ring power train. 


\title{
Laboratory-Model Integrated-System FARAD Thruster
}

\author{
Kurt A. Polzin* \\ NASA-Marshall Space Flight Center, Huntsville, AL 35812
}

\author{
M. Frank Rose ${ }^{\dagger}$ and Robert Miller ${ }^{\ddagger}$ \\ Radiance Technologies, Huntsville, AL 35805
}

\begin{abstract}
The design of an electrodeless thruster that relies on a pulsed, RF-assisted discharge and electromagnetic acceleration using an inductive coil is presented. The thruster design is optimized using known performance scaling parameters and experimentally-determined design rules, with design targets for discharge energy, plasma exhaust velocity, and thrust efficiency of $100 \mathrm{~J} / \mathrm{pulse}, 25 \mathrm{~km} / \mathrm{s}$, and $50 \%$, respectively. Descriptions of the propellant injection, preionzation, and plasma acceleration subsystems that comprise the experimental unit serve to update the design presented in a previous publication. Propellant is injected using a high-speed gas valve and directed over the thruster's face by a conical distribution structure. A high-voltage, pulsed-RF signal preionizes the propellant and permits low-energy current sheet formation and acceleration when the main current pulse passes through the inductive acceleration coil. Electrical measurements are presented to demonstrate operation of each subsystem, and a photograph of the discharge shows the formation of a plasma over the acceleration coil face.
\end{abstract}

\section{Nomenclature}

$\begin{array}{ll}C & \text { capacitance, } \mathrm{F} \\ I & \text { current, } \mathrm{A} \\ \mathrm{ID} & \text { inner diameter, } \mathrm{m} \\ L_{0} & \text { stray inductance, } \mathrm{H} \\ L_{C} & \text { coil inductance, } \mathrm{H} \\ m_{\text {bit }} & \text { mass bit, kg/pulse } \\ \mathrm{OD} & \text { outer diameter, } \mathrm{m} \\ t & \text { time, } \mathrm{s}\end{array}$

C capacitance, $\mathrm{F}$

I current, A

ID inner diameter, $\mathrm{m}$

$L_{0} \quad$ stray inductance, $\mathrm{H}$

$L_{C} \quad$ coil inductance, $\mathrm{H}$

$m_{\text {bit }} \quad$ mass bit, $\mathrm{kg} / \mathrm{pulse}$

$t \quad$ time, $\mathrm{s}$

$\begin{array}{ll}V & \text { voltage, } \mathrm{V} \\ V_{0} & \text { initial charge voltage, } \mathrm{V} \\ v_{z} & \text { exhaust velocity, } \mathrm{m} / \mathrm{s} \\ z & \text { axial distance, } \mathrm{m} \\ z_{0} & \text { electromagnetic decoupling distance, } \mathrm{m} \\ \alpha & \text { dynamic impedance parameter } \\ \eta_{t} & \text { thrust efficiency, } \%\end{array}$

$V \quad$ voltage, $\mathrm{V}$

initial charge voltage, $\mathrm{V}$

exhaust velocity, $\mathrm{m} / \mathrm{s}$

axial distance, $\mathrm{m}$

dynamic impedance parameter

\section{Introduction}

$\mathrm{T}$ HE Faraday Accelerator with Radio-frequency Assisted Discharge (FARAD) ${ }^{1}$ is a type of pulsed inductive plasma accelerator being developed for spacecraft propulsion. The device is electrodeless, with capacitively-stored energy discharged through an inductive coil to induce a plasma near the coil face. The plasma is expelled at high exhaust velocity $[\mathcal{O}(10 \mathrm{~km} / \mathrm{s})]$ by the Lorentz body force arising from the interaction of the plasma current and the induced magnetic field.

Inductive plasma accelerators are attractive as propulsive devices for many reasons. The lifetime and contamination issues associated with electrode erosion in conventional pulsed plasma thrusters (PPTs) do not exist in devices where the discharge is inductively driven. In addition, a wider variety of propellants (e.g. $\mathrm{CO}_{2}, \mathrm{H}_{2} \mathrm{O}$, $\mathrm{NH}_{3}$ ) becomes available for use when compatibility with metallic electrodes is no longer an issue. Moreover, pulsed inductive accelerators (indeed, pulsed accelerators in general) can maintain the same specific impulse and efficiency over a wide range of input power levels by adjusting the pulse rate. A recent review of pulsed inductive thruster technologies can be found in Ref. [2].

FARAD is similar to another device, the Pulsed Inductive Thruster (PIT) ${ }^{3}$ in that both use current flowing through an inductive coil to accelerate a plasma. In the PIT, both propellant ionization and acceleration are performed by the

\footnotetext{
*Propulsion Research Engineer, Propulsion Research and Technology Applications Branch, Propulsion Systems Department. Senior Member AIAA.

$\dagger$ V.P. of Research, Auburn Office, 231 Leach Science Center, Auburn University, Auburn, AL 36849. Associate Fellow AIAA.

${ }^{\ddagger}$ Project Manager, Auburn Office, 231 Leach Science Center, Auburn University, Auburn, AL 36849. Member AIAA.

This material is declared a work of the U.S. Government and is not subject to copyright protection in the United States.
} 
pulse of current in the inductive coil. The PIT MkVa, which presently represents the state-of-the-art in pulsed inductive plasma acceleration, possessed a $1 \mathrm{~m}$ diameter coil and at a discharge energy of $4 \mathrm{~kJ} /$ pulse demonstrated high efficiency ( $\sim 50 \%$ operating on ammonia). In contrast, FARAD uses a separate inductive discharge to preionize the propellant before it is accelerated by the current pulse. ${ }^{1}$ The use of a separate preionization mechanism permits the formation of an inductive current sheet at much lower discharge energies and voltages than those used in the PIT, ${ }^{1}$ with a current sheet being formed even in poorly optimized, low-power ( $44 \mathrm{~J} /$ pulse) proof-of-concept experiments. Relieving the high-energy, high initial voltage design constraint on pulsed inductive current sheet formation can potentially lead to smaller, more compact thrusters for spacecraft systems.

A $100 \mathrm{~J} /$ pulse laboratory-model FARAD thruster was previously presented in Ref. [4]. The thruster subsystems (mass injection, preionization, and acceleration) are integrated into a single unit using a modular design approach that makes it possible to alter or redesign certain aspects of the unit without necessitating refabrication of the entire thruster. Since the initial publication describing this unit, several design changes have been implemented. This paper presents the current thruster design configuration, updating the previous work ${ }^{4}$ to reflect additions and changes to the design. A supporting companion publication ${ }^{5}$ presents data obtained from this iteration of the FARAD thruster.

The outline for the rest of this paper is as follows. In Sect. II, the various subsystems comprising the thruster are presented. Sample data are also presented in this section to demonstrate operation of the thruster's subsystems. Finally, in Sect. III there is a discussion of the triggering and operation of the integrated thruster system.

\section{Thruster Design}

The thruster was designed and optimized based on a number of performance scaling and systems considerations. Detailed reviews of the scaling arguments and design considerations can be found in Refs. [4, 6-8]. In the lab-model FARAD thruster, the inductive coil and its associated drive circuit are optimized for both plasma acceleration and current sheet formation. We assumed a discharge energy of $100 \mathrm{~J} /$ pulse and fixed the acceleration coil dimensions with an inner diameter of $12 \mathrm{~cm}$ and an outer diameter of $30 \mathrm{~cm}$. Optimization led to a set of FARAD thruster target design parameters, which are summarized in Table 1.

In the present FARAD thruster iteration (Fig. 1), there are three major subsystems: propellant injection, preionzation, and acceleration. These subsystems are shown in the figure and each are discussed in turn below. Several of the components discussed below have been redesigned and represent a deviation from the original design presented in Ref. [4].

\section{A. Propellant Injection}

The gas injection system uses a high speed valve (Fig. 2A) with a radial orifice capable of generating gas pulses as short as $70 \mu$ s in pulse width. This valve employs a low mass beryllium copper Belleville spring diaphragm that lifts off the seal at its outer diameter permitting gas to flow

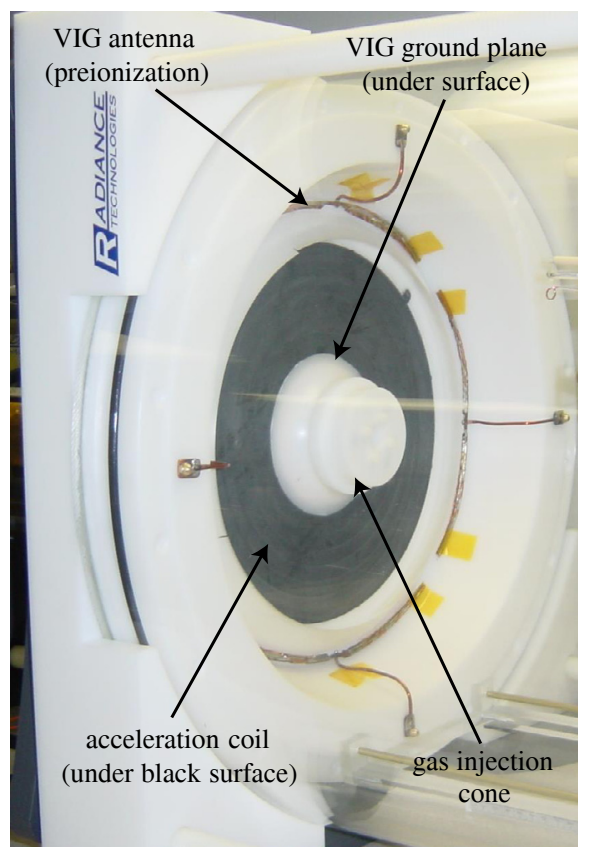

Figure 1. Photograph of the FARAD laboratorymodel thruster coupled to a vacuum chamber.

Table 1. Summary of target FARAD thruster design parameters (terms as defined in the nomenclature).

\begin{tabular}{ll|ll}
\hline \hline$v_{z}$ & $25 \mathrm{~km} / \mathrm{s}$ & $L_{0}$ & $70 \mathrm{nH}$ \\
$\eta_{t}$ & $50 \%$ & $L_{C}$ & $700 \mathrm{nH}$ \\
Energy & $100 \mathrm{~J} /$ pulse & $z_{0}$ & $3.7 \mathrm{~cm}$ \\
$m_{\text {bit }}$ & $150 \mu \mathrm{g}$ (argon) & $C_{e q}$ & $10 \mu \mathrm{F}$ \\
$\alpha$ & 3 & $V_{0}$ & $\geq 3150 \mathrm{~V}$ \\
Coil ID & $12 \mathrm{~cm}$ & $d I / d t$ & $\geq 45 \mathrm{kA} / \mu \mathrm{s}$ \\
Coil OD & $30 \mathrm{~cm}$ & & \\
\hline \hline
\end{tabular}




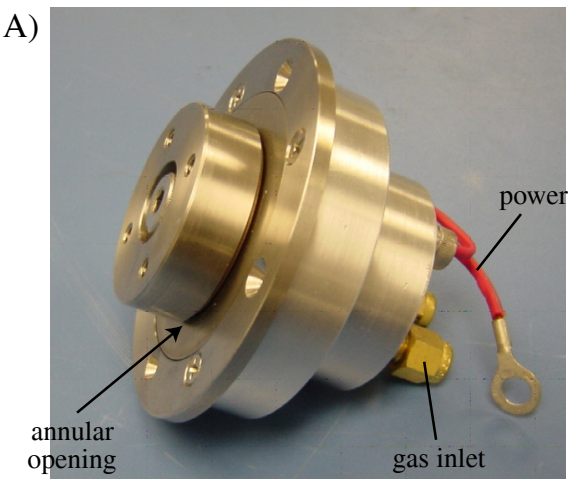

B)

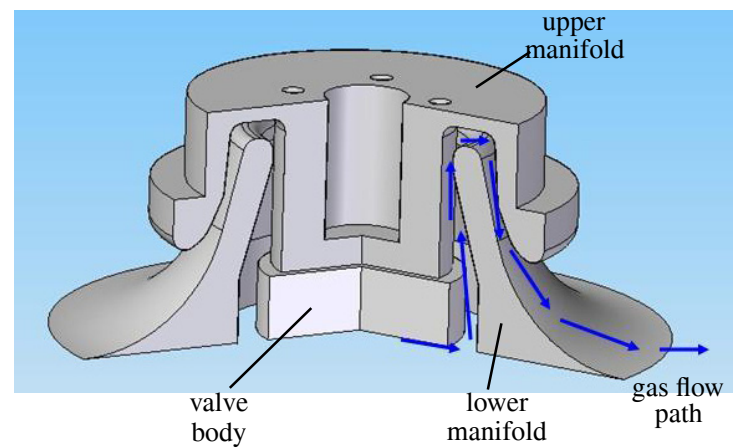

Figure 2. A) High speed gas injection valve. B) Rendering of the gas distribution manifold (shown sectioned to reveal inner detail).

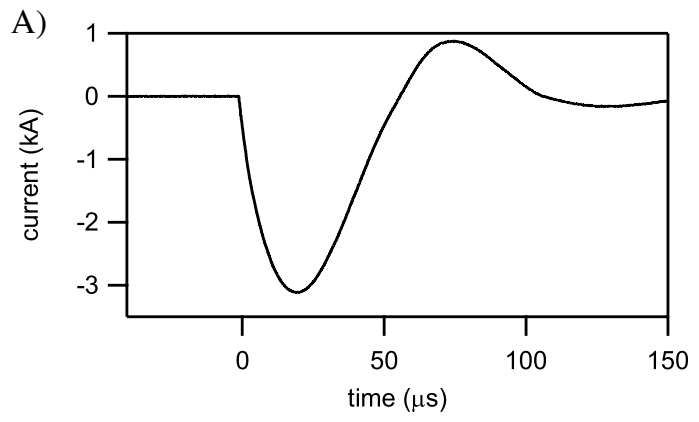

B)

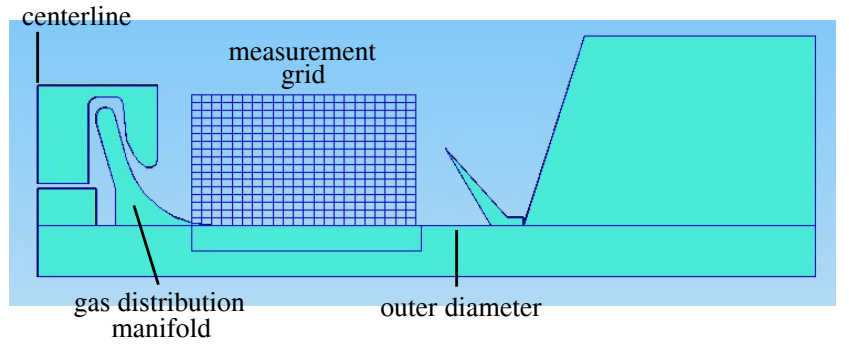

Figure 3. A) Fast gas valve current waveform. B) Measurement grid for fast ionization gauge data.

radially from the valve when open. The diaphragm is driven by a pancake solenoid, which is powered by a solid-state switched $10 \mathrm{kA}, 3 \mathrm{kV}$ pulse generator. The current pulse used to open the valve is presented in Fig. 3A. Continuous mode operation at $5 \mathrm{~Hz}$ or burst-pulse mode operation for 50 pulses at $100 \mathrm{~Hz}$ can be achieved without forced cooling. The expected lifetime of the valve is $10^{8}$ pulses, which is still too low for a real mission of interest, ${ }^{9}$ but useful for work development of a lab-model thruster.

The propellant is distributed over the acceleration coil using the conical manifold shown in Fig. 2B. The temporallyvarying gas distribution over the coil face was measured directly using an Applied Pulsed Power fast ionization gauge (FIG). The instrument was mounted on a translation stage that permitted motion in the $r$ - $z$ plane. The gauge samples a volume with dimensions of $4 \mathrm{~mm}$ in $r, 3 \mathrm{~mm}$ in $\theta$, and $3 \mathrm{~mm}$ in $z$. Prior to use, the FIG was calibrated by varying the argon gas pressure in the chamber constant and measuring these values using an independent gauge. Using the ideal gas law, the gas density corresponding to a given pressure measurement was determined.

Data were obtained over a grid measuring $88 \mathrm{~mm}$ in $r$ and $51 \mathrm{~mm}$ in $z$ (see Fig. 3B). The data collected using the FIG are presented for four different values of $\Delta t$ (measured relative to the start of the valve trigger pulse) in Fig. 4 . These data provide a global picture of the evolution of the gas distribution over the thruster coil face. Unfortunately, there does not appear to be a 'best time' during the gas injection phase to pulse current through the acceleration coil. Figure 5 provides a quantitative illustration of this, showing that early in time a significant fraction of propellant is trapped in the manifold and unavailable for acceleration. However, as time elapses more propellant moves axially away from the thruster face $(z>3 \mathrm{~cm})$, becoming unavailable for propulsive use as it exits the spatial region where electromagnetic acceleration occurs. This is a signnificant issue for thrusters of this class, a represents a major challenge for future design iterations.

\section{B. Preionization}

An antenna assembly is used to capacitively couple energy into the propellant for the purpose of creating the preionized plasma. The antenna consists of a ground plane located at the base of the gas injection manifold and a high voltage conducting ring comprised of 4 segments and located at the outer diameter of the acceleration coil (see Fig. 1). A ring shape was chosen to maintain an azimuthally-uniform electric field over the acceleration coil. 
A)

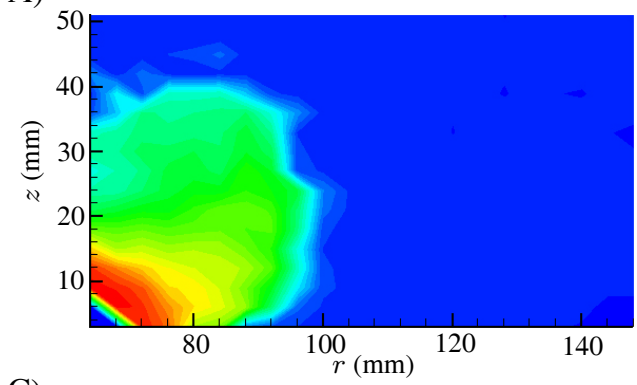

C)

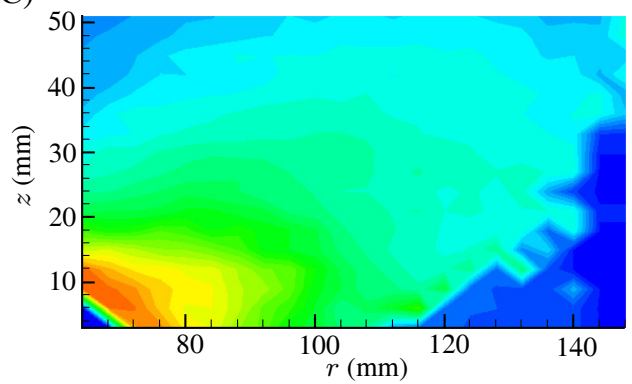

B)

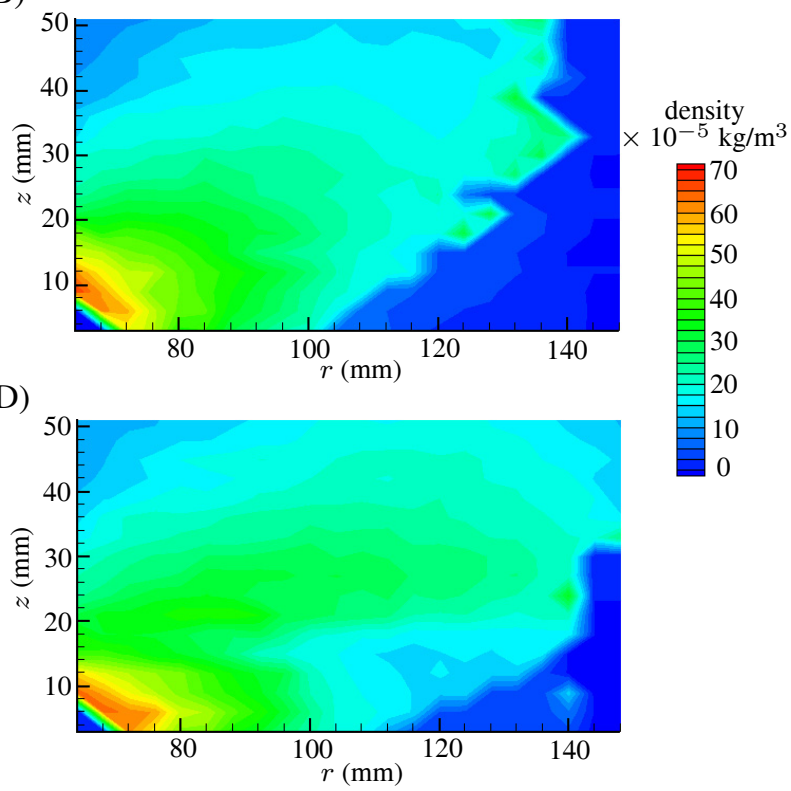

Figure 4. Measured propellant density contours in the thruster at A) $t=0.4 \mathrm{~ms}$, B) $t=0.6 \mathrm{~ms}$, C) $t=0.8 \mathrm{~ms}$, and D) $t=1.0 \mathrm{~ms}$.

The antenna is connected to a vector inversion generator (VIG), which supplies energy in the form of a high-voltage, pulsed-RF signal. The spiral elements of the VIG are shown schematically in Fig. 6A and 6B, and a VIG potted in castrol oil is shown in Fig. 6C. The lines shown in the schematics are metallic foils and the space between them is dielectric film. In this spiral configuration, the VIG consists of two parallel-plate transmission lines wound on a mandrel and sharing a common conductor. During operation, the array is charged to approximately $2 \mathrm{kV}$ with energy stored in the capacitance of the spiral windings, $C_{\text {vig. }}$. The VIG discharge sequence is initiated by closing the input switch, $\mathrm{S}_{1}$.

When switch $S_{1}$ closes, an electromagnetic wave begins traveling along one of the strip lines. As a first-order approx-

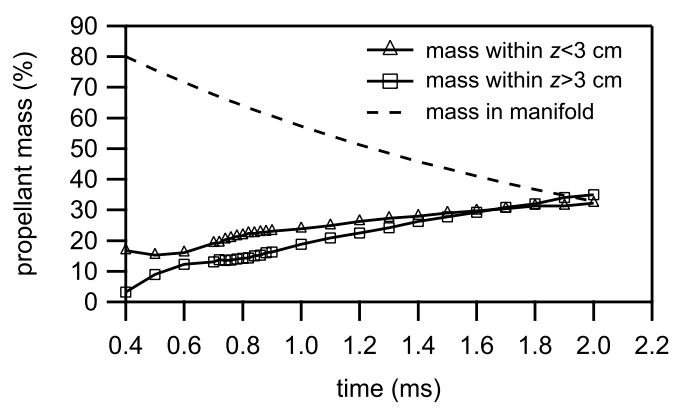

Figure 5. Estimate of propellant mass location as a function of time.
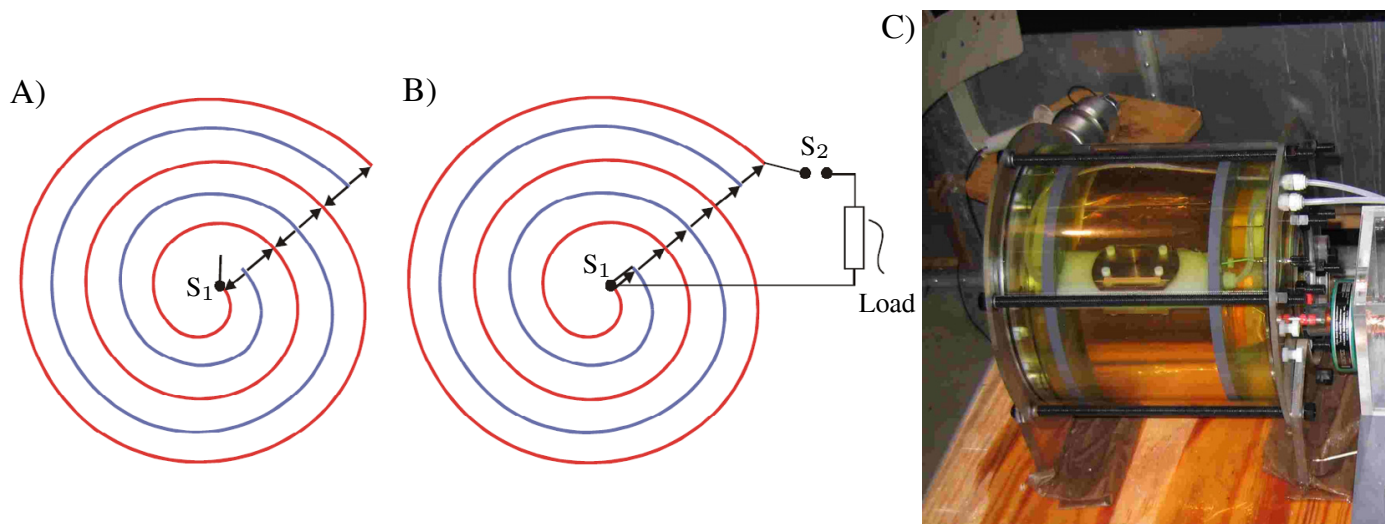

Figure 6. Schematic of a spiral line VIG A) DC charged and B) with electric field vectors all oriented in the same direction. Solid lines are metal foils and the intermediate spaces are insulators. Arrows represent the electric field vectors in each configuration. C) A VIG potted in castrol insulating oil. 
imation, due to the inductance disparity between the two conductors, a wave does not propagate as quickly in the second line. As such, the VIG is comprised of two LC circuits - one "fast" and one "slow". The electric field vectors plotted in Fig. 6A transition to the configuration shown in Fig. 6b after the "fast" wave travels to the "open end" and back to switch $S_{1}$. If the transit time in the fast circuit is sufficiently short when compared to the slow circuit, the voltage across the array will be close to the ideal output of $2 \beta n V_{0}$, where $\beta$ is the efficiency, $V_{0}$ is the initial charge voltage and $n$ is the number of turns in the VIG. The net result is a transient high voltage between the lines at $\mathrm{S}_{1}$ and the outermost turn on the VIG. Voltage outputs as great as $95 \%$ of ideal have been realized in certain geometries.

When the VIG is discharged, the oscillating voltage generates a time varying $E$-field between the outer and the inner antenna rings in the thruster. This partially ionizes the neutral propellant covering the acceleration coil face. The preionization pulse produces a visible, diffuse corona at the base of the gas distribution manifold. While no light emission is observed across the thruster face, the preionized plasma produced by the VIG is necessary for the acceleration coil to consistently break down the propellant and form a current sheet. A sample waveform of the VIG voltage is presented in Fig. 7, demonstrating the high output voltage realized for a charge voltage of only $-1.8 \mathrm{kV}$. The discharge energy for this pulse is approximately $0.1 \mathrm{~J}$, representing a very small portion of the thruster's total energy budget.

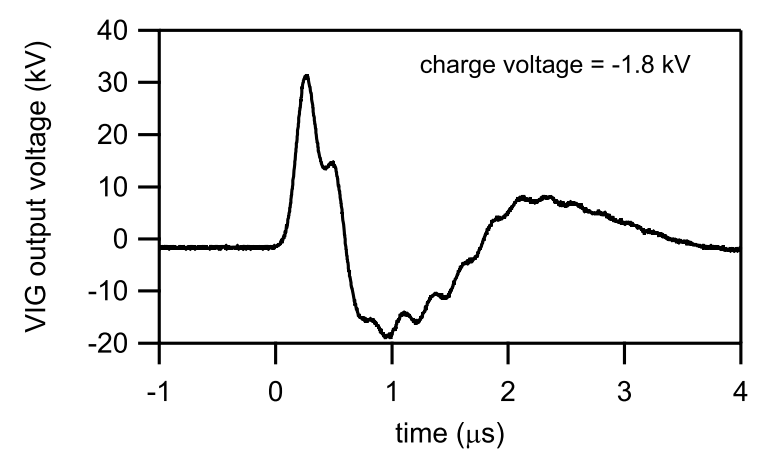

Figure 7. VIG voltage waveform starting at an initial VIG charge voltage of $-1.8 \mathrm{kV}$.

\section{Plasma Acceleration}

In FARAD, the subsystem that performs plasma acceleration is comprised of the acceleration coil, the power train supplying current to the coil, and an electrical transmission line connecting the power train and the coil. Each of these are discussed in turn below.

The most difficult electrical component to design in the pulse circuit is the acceleration coil. Its lumped-element inductance value $L_{C}$ appears in the performance scaling relations, ${ }^{4,6}$ but for a coil consisting of multiple spiral windings, this value cannot be computed analytically. We instead used a 2-D axisymmetric computational modeling method to iteratively adjust the coil design and calculate its inductance. ${ }^{8}$ Once the coil was fabricated, this computational method was validated by comparing the computed inductance profiles, given as a function of current sheet axial position, with experimentally measured profiles. The experimental measurements were obtained using a simulated "current sheet" consisting of a conductive aluminum disk. The inductance was measured using an Agilent 4285A precision LCR meter operating at $100 \mathrm{kHz}$.

The acceleration coil consists of 6 two-turn spirals, with the first complete turn spiraling in from the outer to the inner radius and the second turn spiraling back out to the outer radius. Photographs of the coil, both before and after the coil windings were potted into the thruster assembly, are shown in Fig. 8. The coil path is cut into a base fabricated from Delrin, which has low out-gassing characteristics compared to other plastics that could have been chosen for
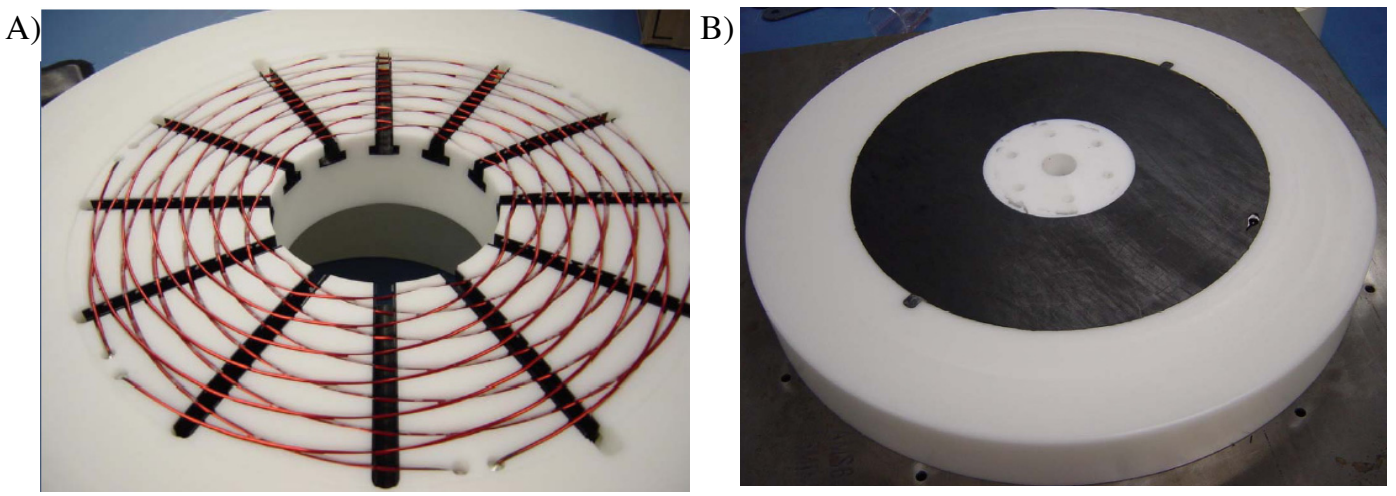

Figure 8. Photographs of the acceleration coil A) before potting and B) after potting. 
the application. Insulated wires are wound into the paths to form the coil. The entire assembly is potted in epoxy to insulate the coil conductors from the inductive plasma.

The measured inductance presented to the circuit as a function of current sheet position followed the well-known empirical scaling

$$
L(z)=L_{0}+L_{C}\left[1-\exp \left(-z / z_{0}\right)\right]
$$

where $L$ is the total inductance measured in the circuit, $L_{0}$ is the stray inductance, and $z_{0}$ is the electromagnetic decoupling length. For the FARAD coil the inductance is $810 \mathrm{nH}$ and the decoupling length is $3 \mathrm{~cm}$. The inductance profiles (both measured and calculated) are given in Fig. 9, demonstrating excellent quantitative agreement. The measurements were performed in a relatively isolated environment, and interactions both between different thruster components or between the fully-assembled thruster and the external environment may serve to reduce the overall inductance of the thruster below the measured level.

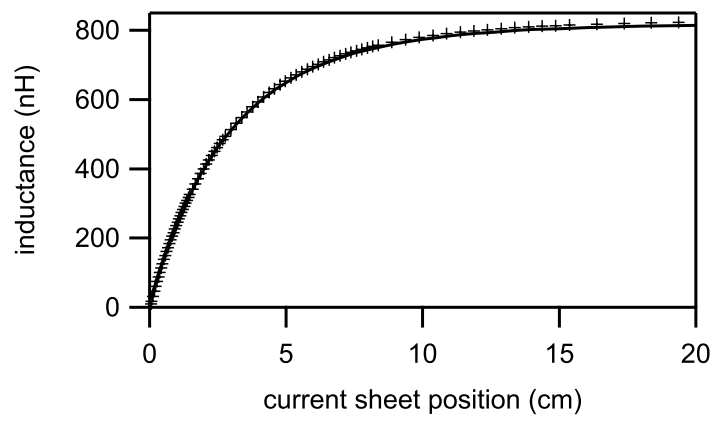

Figure 9. Inductance presented to the circuit by the acceleration coil as a function of current sheet position.

B)
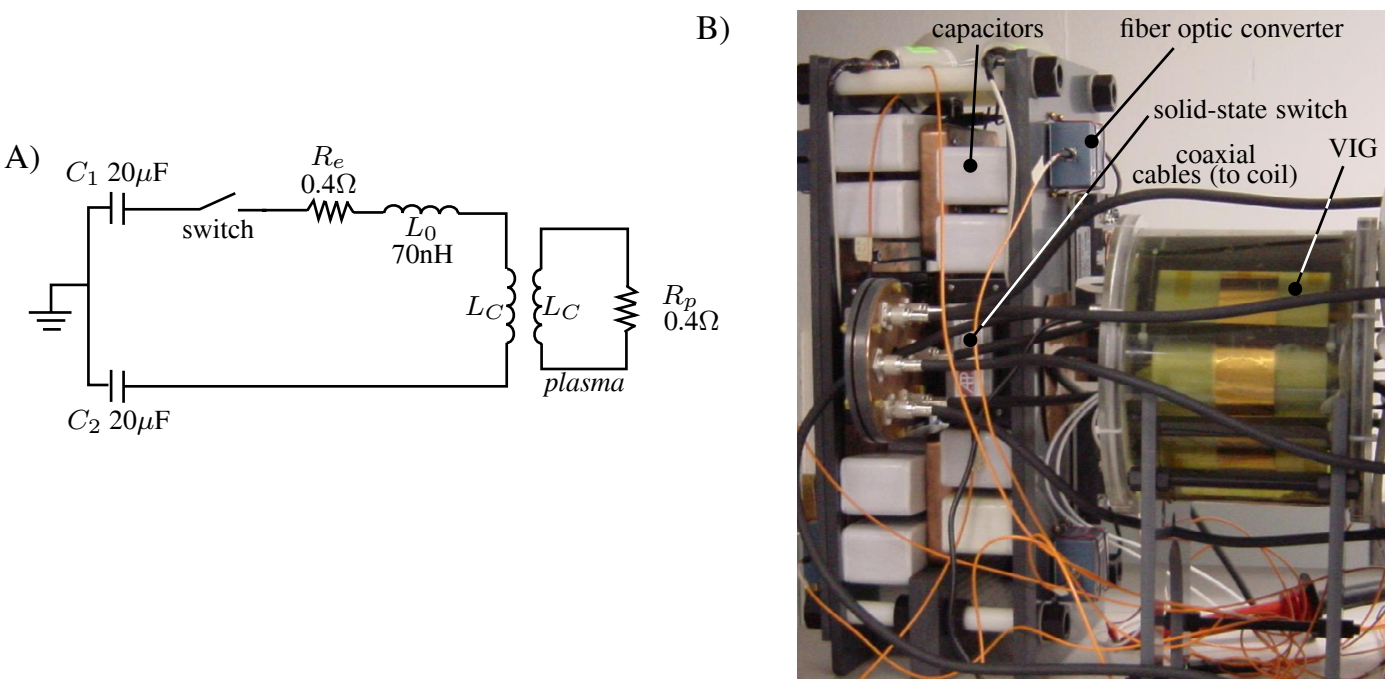

Figure 10. A) Circuit schematic and B) photograph of the B\&M pulse powertrain (shown with the VIG).

The power train used for these initial FARAD experiments was a pulsed Bernardes and Merryman (B\&M) circuit, ${ }^{10}$ which allows unused energy to be recaptured in the charging capacitors. The basic B\&M circuit is shown schematically in Fig. 10A, and a photograph of the B\&M circuit used in the current version of FARAD is shown in Fig. 10B. The circuit has two capacitor banks of equal capacitance. The B\&M is switched using a solid-state switch manufactured by Applied Pulsed Power. When the switch is closed, charge oscillates between the two capacitor banks through the drive coil, represented in the schematic as N1. At the end of the transient the voltage and energy on capacitor banks $C_{1}$ and $C_{2}$ are equal. In this scheme, capacitor bank $C_{1}$ can be brought back to full charge for the next pulse. Meanwhile, bank $C_{2}$ must be discharged before the next pulse is initiated. This setup allows for recovery of at least half the energy remaining after the pulse transient. The target capacitance of $10 \mu \mathrm{F}$ required for acceleration optimization (see Table 1 ) is given by the series configuration where both banks $C_{1}$ and $C_{2}$ are $20 \mu \mathrm{F}$. While the circuit dynamics can be calculated based on a capacitance of $10 \mu \mathrm{F}$, the energy stored for each pulse is equal to $1 / 2\left(C_{1}\right) V_{0}^{2}$. At $20 \mu \mathrm{F}$, the target energy of $100 \mathrm{~J} /$ pulse is obtained for a charge voltage of $3160 \mathrm{~V}$.

The acceleration coil is connected to the B\&M circuit through six parallel coaxial cables. The parallel configuration helps lower the inductance of the connection.

Example current and voltage waveforms for the B\&M circuit are presented in Fig. 11. These measurements demonstrate an additional advantage of the B\&M circuit, namely that it precludes voltage reversal on either capacitor bank. This reduces the stress on the capacitors and increases their operational lifetimes. 


\section{Thruster Operation}

Thruster operation is accomplished by initiating a series of finite-length pulses to actuate and terminate operation of the controllable subsystems. Presented in Fig. 12A is a typical timeline showing the relative times when each of the pulsed systems is switched-on and switched-off. The capacitor bank is charged over a period of $40 \mathrm{~ms}$ when the charging power supply is enabled and an IGBT switch between the supply and the bank is closed (current flowing). At $40 \mathrm{~ms}$, the IGBT switch is opened, disconnecting the bank from the charging power supply, and the gas valve is activated to inject propellant over the coil face. After an additional $1 \mathrm{~ms}$, the VIG and B\&M are triggered, ionizing and accelerating the neutral propellant.

The timing is controlled using a digital delay generator with fiber optic outputs. The optical signals are transformed into electrical control signals using batterypowered, RF-shielded fiber optic converter boxes that are co-located with the pulsed components.

The time-integrated light emission from the thruster operating at $100 \mathrm{~J} /$ pulse is shown in Fig. 12B. The image was obtained using a camera with a framing rate of $1 \mathrm{kHz}$ and an exposure time longer than the discharge. A narrow-pass line filter (488 nm, $10 \mathrm{~nm}$ full-width half-maximum) was used to exclude light not emitted by argon ions. The corona from the VIG discharge is too dim and short-lived to be captured (even without the line filter). Consequently, all the light emission in this photograph is from the plasma induced and coupled to the main current pulse in the acceleration coil. At this early stage of development, no thrust or efficiency measurements have been obtained.

A)
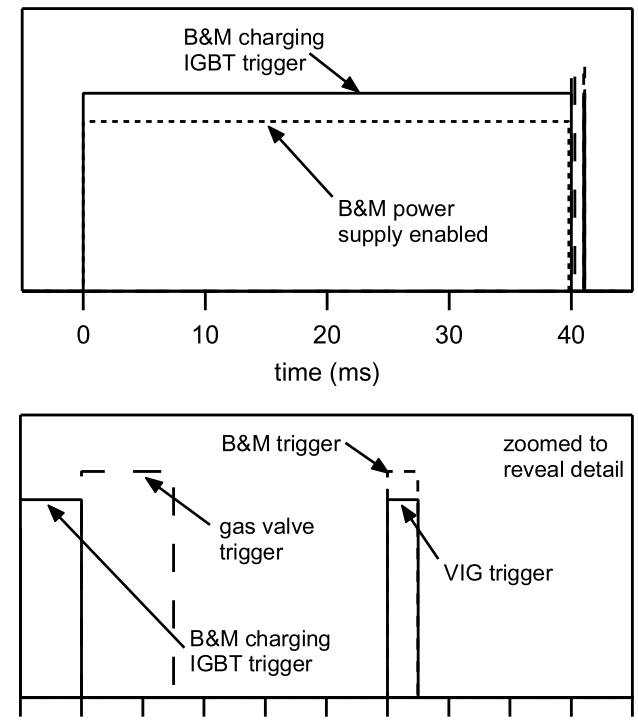

39.840 .040 .240 .440 .640 .841 .041 .241 .441 .641 .8

time $(\mathrm{ms})$

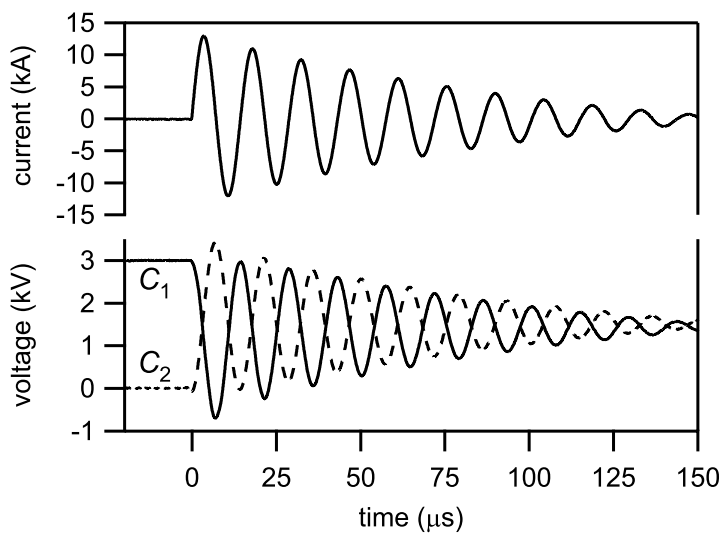

Figure 11. B\&M current and voltage waveforms into a $540 \mathrm{nH}$ inductive load for a $3 \mathrm{kV}$ initial charge on capacitor $C_{1}$. Voltages presented for both capacitors $C_{1}$ and $C_{2}$.

Figure 12. A) Schematic showing the trigger sequencing in the FARAD thruster. B) Time-integrated argon ion light emission (488 nm line) from a single shot of the FARAD thruster operating at a discharge energy of $100 \mathrm{~J}$. 


\section{Conclusions}

We have presented the design of a complete FARAD laboratory-model thruster that relies on a pulsed, RF-assisted discharge and electromagnetic acceleration using an inductive coil. This updates the design presented in a previous publication ${ }^{4}$ and accompanies a companion publication containing experimental data ${ }^{5}$ from this iteration of the FARAD thruster. The thruster is designed to operate at a discharge energy of $100 \mathrm{~J} /$ pulse and is optimized for an exhaust velocity and thrust efficiency of $25 \mathrm{~km} / \mathrm{s}$ and $50 \%$, respectively. A high-speed valve is used to inject propellant and a conical distribution structure serves to spread the neutral gas over the coil face. A pulsed-RF signal produced from a vector inversion generator is coupled into the gas using an antenna structure, preionizing the propellant before the main discharge. A high-current pulse through the acceleration coil couples to the preionized plasma, further ionizing and electromagnetically accelerating the propellant. The data are presented to describe the operation of each subsystem in isolation from the others, and time-integrated photography indicates the presence of a relatively dense plasma forming over the acceleration coil face when all subsystems are operated in concert.

\section{Acknowledgments}

This work was performed under NASA contract NNM06AA17G, managed by Dr. Michael LaPointe, supporting the West Virginia High Technology Consortium Foundation's Pulsed Plasma Accelerator (PPA06) program.

\section{References}

\footnotetext{
${ }^{1}$ E.Y. Choueiri and K.A. Polzin, "Faraday Acceleration with Radio-frequency Assisted Discharge," J. Propuls. Power, 22(3):611, May-June 2006.

${ }^{2}$ K.A. Polzin, "Comprehensive Review of Planar Pulsed Inductive Plasma Thruster Research and Technology," J. Propuls. Power, submitted for publication, July 2008 .

${ }^{3}$ C.L. Dailey and R.H. Lovberg, The PIT MkV Pulsed Inductive Thruster, Tech. Rep. NASA CR-191155, TRW Systems Group, July 1993.

${ }^{4}$ K.A. Polzin, M.F. Rose, R. Miller, S. Best, T. Owens, and J. Dankanich, "Design of a Low-Energy FARAD Thruster," 43rd AIAA/ASME/SAE/ASEE Joint Propuls. Conf., Cincinnati, OH, July 2007. AIAA Paper 2007-5257.

${ }^{5}$ K.A. Polzin, M.F. Rose, and R. Miller, "Operational Characteristics and Plasma Measurements in a Low-Energy FARAD Thruster," 44th AIAA/ASME/SAE/ASEE Joint Propuls. Conf., Hartford, CT, July 2008. AIAA Paper 2008-5011.

${ }^{6}$ K.A. Polzin and E.Y. Choueiri "Performance Optimization Criteria for Pulsed Inductive Plasma Acceleration," IEEE Trans. Plasma Sci., 34(3):945, 2006

${ }^{7}$ K.A. Polzin, "Scaling and Systems Considerations in Pulsed Inductive Plasma Thrusters," 30th Internat'l Electric Propuls. Conf., Florence, Italy, Sept. 2007. IEPC Paper 2007-192.

${ }^{8}$ K.A. Polzin, "Scaling and Systems Considerations in Pulsed Inductive Plasma Thrusters," IEEE Trans. Plasma Sci., In Press, Aug. 2008.

${ }^{9}$ J.W. Dankanich and K.A. Polzin, "Mission Assessment of the Faraday Accelerator with Radio-frequency Assisted Discharge (FARAD)," 44th AIAA/ASME/SAE/ASEE Joint Propuls. Conf., Hartford, CT, July 2008. AIAA Paper 2008-4517.

${ }^{10}$ J. Bernardes and S. Merryman, "Parameter Analysis of a Single Stage Induction Mass Driver," Proc. 5th IEEE Int'l Pulsed Power Conf., M.F. Rose and P.J. Turchi, ed., pp. 552-555, Arlington, VA, 1985. Paper PI-27.
} 